\title{
Liquorice-induced apparent mineralocorticoid excess presenting in the emergency department
}

\author{
Authors: Stuart Declan Gallacher, ${ }^{A}$ Georgios Tsokolas $^{B}$ and Ioannis Dimitropoulos ${ }^{C}$
}

\begin{abstract}
A 65-year-old woman with a background of myalgic encephalitis, who was taking alternative medicines and dietary supplements, presented with hypokalaemia and hypertension. After a thorough history it became apparent that this was most likely secondary to regular consumption of liquorice tea. The patient was advised to discontinue drinking this tea and was discharged. Follow-up showed normalising blood pressure and hypokalaemia, with a normal aldosterone:renin ratio.
\end{abstract}

KEYWORDS: 11ß-hydroxysteroid dehydrogenase, apparent mineralocorticoid excess, complementary and alternative medicine, glycyrrhizic acid, liquorice

\section{Case presentation}

A 65-year-old woman presented to our emergency department with a 1-day history of increasingly frequent paroxysmal palpitations; episodes lasted around 1 minute and were associated with feeling persistently faint and tired. She had consulted her GP earlier that day, who noted her to be hypokalaemic and advised her to present to hospital for investigation.

On admission, her serum potassium was $2.4 \mathrm{mmol} / \mathrm{L}$, her blood pressure was 198/127 mmHg, and an electrocardiogram showed ventricular bigeminy with a rate of $70 \mathrm{bpm}$. Clinical examination and chest X-ray were unremarkable. Her past medical history was significant for myalgic encephalitis and she admitted to taking two homeopathic medications (argentum nitricum and Aconitum napellus), neither of which are known to exert any pharmacological effect. She was also taking magnesium and oral multivitamins as a form of self-medication for persistent symptoms of myalgic encephalitis. She was taking no other regular medication. She was treated initially with 2 bags of $500 \mathrm{~mL} 0.9 \%$ sodium chloride solution with $40 \mathrm{mmol}$ of potassium in each and was observed on continuous cardiac monitoring overnight with a presumptive diagnosis of Conn's syndrome.

Authors: ${ }^{A}$ FY2 doctor, Plymouth Hospitals NHS Trust, Plymouth, UK; ${ }^{B}$ FY2 doctor, Mid Cheshire Hospitals NHS Foundation Trust, Crewe, UK; ${ }^{C}$ consultant endocrinologist, Plymouth Hospitals NHS Trust, Plymouth, UK
On direct questioning it became apparent that the patient also drank liquorice tea three times a day. She was advised of the potentiating effect liquorice can have on the function of cortisol, its relevance to her symptoms and that she should discontinue drinking it. Given this possible explanation for the apparent mineralocorticoid excess (AME), a symptomatic improvement of her palpitations and the improvement in her potassium level, she was discharged with 3 days of oral potassium supplementation.

\section{Differential diagnosis}

On initial presentation, it was felt that the most likely diagnosis would be Conn's or Liddle's syndrome. However, an expedient and thorough history of alternative medications and relevant dietary intake quickly established a reasonable alternative diagnosis. The rapid and persistent normalisation of potassium and blood pressure after discharge and the normal aldosterone:renin ratio are all consistent with liquoriceinduced AME rather than Conn's syndrome.

\section{Initial management and prognosis}

Initial therapy was directed at correcting the biochemical abnormality that had prompted the acute presentation to hospital. Once potassium was established at a safe level and appeared to be stable, it was felt that no immediate further treatment would be required. The aetiology was quickly established and definitive treatment simply involved avoidance of liquorice tea. The overall prognosis is excellent with complete and sustained improvement in symptoms, biochemical abnormalities and hypertension expected rapidly after withdrawal of the liquorice preparation.

\section{Case progression and outcome}

Her potassium level normalised over the course of the next week and a follow-up aldosterone:renin ratio 3 months later was normal (Table 1). Blood pressure had improved to 152/83 mmHg and she had suffered no further palpitations. The patient was discharged with no further follow-up planned.

\section{Discussion}

AME, including chronic liquorice ingestion, is characterised by hypokalaemia, hypertension, metabolic alkalosis, a 
Table 1. Biochemistry investigations

\begin{tabular}{lllll} 
Biochemistry results & Admission & Discharge & $\begin{array}{l}\text { Follow-up 13 days post- } \\
\text { discharge }\end{array}$ & $\begin{array}{l}\text { Endocrine follow-up } 3 \text { months } \\
\text { after discharge }\end{array}$ \\
Sodium (133-146 mmol/L) & 145 & 149 & 142 & 4.6 \\
Potassium $(3.5-5.3 \mathrm{mmol} / \mathrm{L})$ & 2.4 & 3.1 & 62 & 3.7 \\
Creatinine $(44-80 \mu \mathrm{mol} / \mathrm{L})$ & 69 & 43 & 89 & $<29$ \\
eGFR $\left(\mathrm{mL} / \mathrm{min} / 1.73^{2}\right)$ & 78 & $>90$ & $<7.8$ \\
Supine renin $(2-30 \mathrm{mU} / \mathrm{L})$ & & & \\
Supine aldosterone $(<160 \mathrm{ng} / \mathrm{L})$ & & & \\
Aldosterone:renin ratio & & & \\
\hline Normal values or ranges are given in parentheses. eGFR = estimated glomerular filtration rate &
\end{tabular}

low plasma renin activity and a low plasma aldosterone level. Presentation is often similar to that of Conn's syndrome and the differential diagnosis is based on plasma aldosterone levels. By definition, this is high in Conn's and characteristically low in AME. Other, rare causes for AME should also be considered where there remains diagnostic doubt. Ectopic adrenocorticotropic hormone production, causing increased secretion of non-aldosterone steroids with mineralocorticoid effects, may be considered. Characterised by a greatly elevated 24 -hour urinary free cortisol excretion, high serum ACTH and Cushingoid facies, investigation of the source of the ACTH would be paramount. Liddle's syndrome, an autosomal recessive genetic disorder characterised by increased sodium resorption due to a mutation in the epithelial sodium channel in the collecting tubule of the kidney, may present similarly and would be best investigated via a thorough family history and testing for the characteristic genetic mutation.

$11 \beta$-hydroxysteroid dehydrogenase (11 $\beta$-HSD) exists as a pair of isoenzymes, type $1(11 \beta-\mathrm{HSD} 1)$ catalyses the conversion of metabolically inert cortisone to active cortisol, while type 2 (11/-HSD2) catalyses the opposite reaction. The active ingredient of liquorice, glycyrrhizic acid (GA), and some of its metabolic by-products have previously been identified as inhibitors of $11 \beta$-HSD2 function. This can result in an AME, a longer half-life of plasma cortisol and reduced plasma cortisone. ${ }^{1} 11 \beta-$ HSD is highly expressed in mineralocorticoid target tissues, such as the renal cortex, and is thought to help rapid regulation of mineralocorticoid functions of cortisol, while having no effect on aldosterone activation of the mineralocorticoid receptor. ${ }^{2}$ Previous authors have highlighted that signs of AME are not present with consumption of less that $50 \mathrm{~g} /$ day of liquorice. ${ }^{3}$ However, this case highlights several practical difficulties with this. Firstly, it is, in fact, the level of GA that is significant and this value is not typically available to consumers. Secondly, it is also unknown whether the concentrations or bioavailability of GA vary in different preparations of liquorice.

Little formal guidance exists for management of this condition. However, several case studies highlight that full recovery of signs and symptoms of AME generally occurs quickly on discontinuation of liquorice. ${ }^{4}$ It has also been shown that the ratio of urinary cortisol:cortisone normalises within
2 weeks, even after prolonged ingestion of large quantities of liquorice. ${ }^{5}$ It is less clear what effect liquorice excess has on the renin-angiotensin axis as it has no direct effect on the production of aldosterone. It has previously been shown that activity of the renin-angiotensin axis is reduced in such cases. ${ }^{5}$ This may be due to the inappropriate mineralocorticoid effect exerted by excess cortisol. Typically, this normalises within 4 months of cessation of intake of liquorice.

In the treatment of AME, it is an appropriate course of action to supplement any potassium deficiency and to remove liquorice from the diet entirely, regardless of the quantity or preparation being taken. If the potassium then remains stable and blood pressure improves, patients can be followed up, with measurement of an aldosterone:renin ratio, approximately 4 months after discontinuation of liquorice.

\section{Key learning points}

$>$ Liquorice is a potent and reversible inducer of the mineralocorticoid effects of cortisol.

$>$ A thorough history of alternative medications is advisable in all cases, preparations and concentrations vary wildly and pharmacological effects may echo this.

> Early recognition of alternative medications and dietary supplements prevents unnecessary investigation and distress in presentations of apparent mineralocorticoid excess.

> Withdrawal of dietary liquorice, even after prolonged ingestion or ingestion of very large quantities, is associated with a sustained and complete resolution of the symptoms of induced apparent mineralocorticoid excess.

\section{Conflicts of interest}

The authors have no conflicts of interest to declare.

\section{Acknowledgements}

Informed written consent was obtained from the patient before writing this article.

\section{References}

1 Quinkler M, Stewart PM. Hypertension and the cortisol-cortisone shuttle. J Clin Endocrinol Metab 2003;88:2384-92. 
2 Stewart PM, Murray BA, Mason JI. Human kidney 11-beta-hydroxysteroid dehydrogenase is a high affinity nicotinamide adenine dinucleotide-dependent enzyme and differs from the cloned type 1 isoform. J Clin Endocrinol Metab 1994;79:480-4.

3 van Uum SH. Liquorice and hypertension. Neth J Med 2005;63:11920.

4 de Klerk G, Nieuwenhuis MG, Beutler JJ. Hypokalaemia and hypertension associated with use of liquorice flavoured chewing gum. BMJ 1997;314:731-2.
5 Farese RV, Biglieri EG, Shackleton CHL et al. Liquorice-induced hypermineralocorticoidism. N Engl J Med 1991;325:1223-7.

Address for correspondence: Dr S D Gallacher, Derriford Hospital, Derriford Road, Derriford, Plymouth PL6 8DH, UK. Email: stuartgallacher@nhs.net 